\title{
$\begin{array}{ll}\text { Research Square } & \begin{array}{l}\text { Preprints are preliminary reports that have not undergone peer review. } \\ \text { They should not be considered conclusive, used to inform clinical practice, } \\ \text { or referenced by the media as validated information. }\end{array}\end{array}$
}

\section{Amelioration of Cadmium-induced Oxidative Damage in Wistar Rats by Vitamin C, Zinc and N-Acetylcysteine}

\author{
Venkataramanaiah Poli \\ Sri Venkateswara University \\ Renuka Madduru \\ Sri Venkateswara University \\ Srinivasulu Reddy Motireddy ( $\sim$ pvr9490641036@gmail.com ) \\ Sri Venkateswara University
}

\section{Research Article}

Keywords: Cadmium , Vitamin C , Zinc , N-Acetylcysteine, Oxidative damage , Amelioration potential

Posted Date: August 24th, 2021

DOI: https://doi.org/10.21203/rs.3.rs-775144/v1

License: @ (i) This work is licensed under a Creative Commons Attribution 4.0 International License. Read Full License

Version of Record: A version of this preprint was published at Medical Sciences on January 26th, 2022. See the published version at https://doi.org/10.3390/medsci10010007. 


\section{Abstract}

The present study was performed to determine the protective effects of Vitamin C, Zinc and N-Acetylcysteine individually or in combinations with $\mathrm{Cd}$, to monitor amelioration capability against $\mathrm{Cd}$-induced oxidative damage in Wistar rats. Nine groups of rats were studied as experimental group. The present experiment was conducted for 45 days. Liver and kidneys were excised for biochemical determinations through assaying of antioxidant enzymes and lipid oxidation products to assess the impact of Cdtoxicity and its amelioration by co-administration of Vitamin C, Zinc and NAC along with Cd. Basal Metabolic rates, Tissue Respiration rates of liver and kidney were found to be significantly decreased $(p<0.05)$ during Cd toxicity. Serum biochemical parameters were also found to be significantly altered to cope up with $\mathrm{Cd}$ toxicity. All the antioxidant enzymes and products were significantly inhibited $(p<0.05)$ or elevated in rat liver and kidney tissues during $C d$-induced toxicity. Whereas with co-administration of Vitamin C, Zinc and NAC, into rats clearly demonstrates the amelioration of oxidative damage induced by Cd-toxicity. From the results obtained in the present study all the agents tested had protective effects against Cd-induced oxidative damage.

\section{Introduction}

Metallic elements are intrinsic components of the environment that it is difficult to be removed completely. With the increasing use of a wide variety of metals in industry and in our daily life, serious problems resulting from toxic metal pollution of the environment still have been studied. Heavy metals can be accumulated by organisms through a variety of pathways, including respiration, absorption and ingestion, highly toxic to many organs of both humans and animals [1, 2]. Cadmium (Cd) is one of the most toxic heavy metals. It is used in the manufacture of pigments, stabilizers, alloys, electronic compounds; thus especially in rechargeable Nickel-Cadmium batteries; thus its presence in the atmosphere has increased with industrial development $[3,4]$. Cd as a naturally occurring metal can be found in food, water, and cigarette smoke. However, the mechanism and pathway for the toxic effects of $\mathrm{Cd}$ at tissue level were not yet well understood. Several studies have revealed that Cd-toxicity stimulates the production of reactive oxygen species (ROS) and the induction of oxidative stress in different organs [5]. Moreover, Cd-exposure stimulates lipid peroxidation-induced tissue damage [6]. In living cells, protection against oxidative damage encompasses enzymatic and non-enzymatic antioxidant systems. Impairment of the enzymatic antioxidant system due to abnormal gene expression was mainly attributed to Cd-induced oxidative stress [7]. Disruptions of the cellular oxidation-reduction balance may cause damage to tissues and organs and impair their functions [5]. Currently, research related to improving the antioxidant status after Cd-toxication is of major importance. It is well known that endogenous antioxidants may play an imperative role in antioxidative defense against oxidative impairment, possibly by protecting the biological cells function [5, 8]. Natural antioxidants, vitamins A, D, E, Carotenoids, certain elements are being consumed through diet playing a vital role in maintenance of antioxidant status in biological system. Recently much interest has been given to the natural antioxidants as prevention against oxidative damage as a factor in the pathophysiology of various health issues [9] (Amel et al., 2020). Among a range of antioxidants, Vitamins, Zinc and NAC were found to play an important role in the regulation of the antioxidant system $[8,5]$. Vitamin $\mathrm{C}$ is a water soluble chain-breaking antioxidant and can scavenge superoxide and hydrogen peroxide [10]. Zinc is an essential trace element in human beings, relatively non-toxic ubiquitous in subcellular metabolism and an integral component of catalytic sites of enzyme classification. N-Acetylysteine (NAC) is a small molecule containing a thiol group, which has antioxidant properties and is freely filterable with a ready access to intracellular compartments. The diversity of pharmacological applications of NAC is due, mainly to the chemical properties of the cysteinyl thiol group of its molecule, since the ability of reduced thiol groups to scavenge oxygen free radicals is well established. To date, the full impact of the environmental contamination has not been elucidated in the biological system, where the effect of $\mathrm{Cd}$, either individually or in combinations with natural antioxidants and trace elements require a thorough examination to understand the interaction between Cd with antioxidants. Therefore, this report addresses the oxidative stress marker enzyme that constitute an important part of antioxidant defense mechanism in cellular systems and also examines whether the co-administration of Vitamin C, Zinc, NAC both individually and also in combination, can reverse $\mathrm{Cd}$-induced tissue oxidative damage or biochemical pathways using oxidative marker enzymes as oxidative defense biomarkers.

\section{Materials And Methods}

\section{Animals}


Male rats weighing $225 \pm 10 \mathrm{~g}$ were selected in the present study and were housed in stainless steel mesh cages, under standard laboratory conditions (Temperature $23 \pm 2^{\circ} \mathrm{C}, 50 \pm 10 \%$, Relative humidity: 12:12 Light: Dark cycle). The animals were fed with standard rat chow (obtained from Sai Durga Feeds and Foods, Bangalore) and drinking water ad libitum. The rats were acclimatized to the laboratory conditions for ten days. The Institutional Animal Ethics Committee has approved the Experimental protocols and animal use (Resolution No. 60b/2012/(i)/a/CPCSEA/IAEC/SVU/ MSR-RS dt. 08.07.2012), Sri Venkateswara University, Tirupati, Andhra Pradesh, India.

\section{Chemicals}

Cadmium as Cadmium chloride $\left(\mathrm{CdCl}_{2}\right)$, Zinc as Zinc chloride $\left(\mathrm{ZnCl}_{2}\right)$, Vitamin C (Ascorbic acid) were obtained from Sigma Chemical Co, Loba Chemicals and Sd-Fine chemicals, India. All the chemicals used in this study were of the highest purity.

\section{Experimental Design}

Rats were divided into 9 groups; each contained 6 rats and fed one of the following diets.

Group 1: Control group

Group 2: $\mathrm{CdCl}_{2}$ dissolved in drinking water @ $10 \mathrm{mg} / \mathrm{lit}$

Group 3: $\mathrm{CdCl}_{2}(10 \mathrm{mg} / \mathrm{lit}$ of drinking water $)+$ Vitamin C (100 mg/ kg BW)

Group 4: $\mathrm{CdCl}_{2}(10 \mathrm{mg} / \mathrm{lit}$ of drinking water $)+$ Zinc $(15 \mathrm{mg} / \mathrm{kg})$

Group 5: $\mathrm{CdCl}_{2}(10 \mathrm{mg} /$ lit of drinking water $)+\mathrm{NAC}(100 \mathrm{mg} / \mathrm{kg}$ through gavage $)$

Group 6: $\mathrm{CdCl}_{2}(10 \mathrm{mg} /$ lit drinking water $)+$ Vitamin C $(100 \mathrm{mg} / \mathrm{kg} \mathrm{BW})+$ Zinc $(15 \mathrm{mg} / \mathrm{kg})$

Group 7: $\mathrm{CdCl}_{2}(10 \mathrm{mg} /$ lit drinking water $)+$ Vitamin C (100 mg/kg BW $)+$ NAC (100 mg/kg through gavage)

Group 8: $\mathrm{CdCl}_{2}(10 \mathrm{mg} /$ lit drinking water $)+$ Zinc $(15 \mathrm{mg} / \mathrm{kg})+\mathrm{NAC}(100 \mathrm{mg} / \mathrm{kg}$ through gavage $)$

Group 9: $\mathrm{CdCl}_{2}(10 \mathrm{mg} /$ lit drinking water $)+$ Vitamin C $(100 \mathrm{mg} / \mathrm{kg} \mathrm{BW})+$ Zinc $(15 \mathrm{mg} / \mathrm{kg})+\mathrm{NAC}(100 \mathrm{mg} / \mathrm{kg}$ through gavage $)$

Quantity of food consumed by rat 35-60 g forage/day and of drinking water $25-40 \mathrm{ml} /$ day.

After completing the study, all the groups of animals were accurately weighed and weights were recorded. Animals anesthetized and blood samples were collected through cardiac puncture. Animals were sacrificed by cervical dislocation and the tissues like liver and kidney were isolated and kept at $4^{0} \mathrm{C}$ for further biochemical analysis. Serum samples were separated by using centrifugation at 2000 rpm for 20 minutes. Serum samples were used for biochemical analysis.

\section{Biochemical analyses were performed by following methods}

The organ weights were presented as Relative Organ was calculated as follows

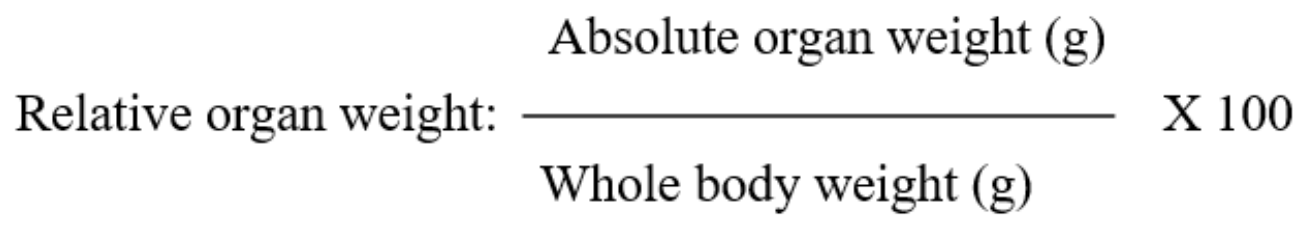




\begin{tabular}{|ll|}
\hline Tissue respiration & Umbriet et al., (1959) [11] \\
\hline Protein & Lowry et al., (1954) [12] \\
\hline Urea & Patton Crouch, (1977) [13] \\
\hline Creatinine & Faulkner et al., (1976) [14] \\
\hline Lactate dehydrogenate & Kornberg (1974) [15] \\
\hline Aspartate Amino transferase & Reitman \& Frankel, (1957) [16] \\
\hline Alanine Amino transferase & Reitman \& Frankel, (1957) [16] \\
\hline Malanoldehyde & Ohkawa et al., (1979) [17] \\
\hline Superoxide dismutase & Misra \& Fridovich, (1972) [18] \\
\hline Catalase & Aebi et al., (1985) [19] \\
\hline Lipid peroxidation & Ohkawa et al., (1979) [17] \\
\hline Glutathione peroxidase & Wendel (1981) [20] \\
\hline Glutathione & Moran et al., (1979) [21] \\
\hline
\end{tabular}

\section{Statistical Analysis and Data Presentation}

Results obtained were presented as Mean \pm SD for comparison of different experimental animal groups with control groups. All the data obtained were statistically analyzed using SPSS package. The results were statistically analyzed by a one way ANOVA. P value $<0.05$ was considered significant. Data of biochemical measurements were further subjected to estimation of percent changes caused by exposure to the Cd with co-administration with Vitamin C, Zinc, NAC individually and also in combinations.

\section{Results}

In the present study nine groups of rats were maintained for a period of 45 days. No mortality occurred during the experimental period.

\section{Body Weights and Relative Tissue Weights}

Initial Body Weights (IBW), Final Body Weights (FBW) and Relative Tissue Weights for Liver and Kidney of male rats subjected to different experimental treatments were obtained and presented in Table.1. The FBW's were significantly decreased $(-45.89 \% ; p<0.05)$ compared to control, whereas for Cd-cotreatment individually with Vitamin C, Zinc or NAC or in combinations recorded FBW's were not significant. Similarly the Body Weight gain or loss also found to be statistically significant $(p<0.05)$ in Cd-administered rats compared to non-significant increase recorded with Cd-co-administered rats with Vitamin C, Zinc or NAC either individually or in combinations which falls in the range of 237 to $238 \mathrm{~g}$. The Body weight gain was found to be around $5 \%$ and was not significant in Cd-co-administered with Vitamin C, Zinc or NAC either individually or in combinations. The individual Liver weights of rats of different experimental groups showed a significant decrease $(p<0.05)$ ranges between 23 to $56 \%$. Maximum decrement $(-55.54 \%)$ recorded with Cd-treated group of rats compared to control group, whereas 23 to $33 \%$ was recorded with other experimental group of rats. Histo-somatic index $(\mathrm{HSI})$ of liver was found to be significantly $(\mathrm{p}<0.05)$ decreased in all the experimental group of rats except $\mathrm{Cd}-$ treated group of rats found to be Not Significant (NS). The relative weights of kidney were also found to be significantly $(p<0.05)$ decreased in all the experimental group of rats. The percent decrease was found to ranges between 27 to $44 \%$ in all the experimental group of rats. The HSI of kidney were found to be significantly decreased $(p<0.05)$ in all the experimental group of rats except $\mathrm{Cd}$ treated group of rats, which was found to be Not Significant (NS).

\section{Respiratory \& Antioxidant Status}


Basal Metabolic Rate (BMR) and Tissue Respiratory (TR) potentials of both liver and kidney were monitored and presented in Table.2. The BMR and TR values were found to be significantly $(p<0.05)$ decreased in all the experimental group of rats compared to control group. The Biochemical parameters of Serum were monitored and presented in Table.3. The Total Protein, Albumin, Urea, Creatinine, Lactate dehydrogenase (LDH), Aspartate amino transferase (AST) and Alanine amino transferase (ALT) were found to be significantly $(p<0.05)$ elevated or increased in all the experimental groups of rats compared to control group of rats. The Antioxidant enzymes and products were estimated in liver and kidney tissues in all the experimental group of rats and presented in Tables.4\&5, respectively. Superoxide dismutase (SOD), Catalase (CAT), Glutathione peroxidase (GPx) and Glutathione (GSH) contents were found to be significantly $(p<0.05)$ decreased in all the groups of experimental rat groups compared to control group of rats. Lipid peroxidation values were significantly $(p<0.05)$ increased in all the groups of experimental rats compared to control group.

\section{Discussion}

\section{Body weights and Relative organ weights}

In the toxicological studies, it has been reported that body and organ weights were considered as an important criterion for the evaluation of organ toxicity. The decrease or increase of body weights during Cd toxicity generally portrays the toxic effects of Cd or xenobiotic. Body weights and relative organ weights of experimental rats i.e. Cd-treated group and also co-administered with vitamin C, Zinc, NAC either individually or combinations were significantly reduced. Immunization evokes lot of pain, distress and inflammation consequently reducing the animals movement and appetite, thereby leading to a significant decrease in food uptake i.e. anorexia or food avoidance or post food palatability due to Cd-toxicity. Cd-induced toxicity involves the induction of oxidative stress results in the alterations in the antioxidant status, leading to severe metabolic disorders and weight loss. Several earlier reports also states that inflammation causes weight loss ranges between 1-20\% due to Cd-induced toxicity or treatment [22]. In the present study Vitamin C, Zinc, NAC are considered to be more efficient in attenuation of Cd-induced toxicity and restore Cd-toxicity including metabolic status, increasing food intake, body weight, organ and body weights to the maximum possible extent.

\section{Respiratory \& Antioxidant Status}

Generally the level of organismic metabolism by measuring the rate of oxygen consumption. It is known that the respiratory rates after under the influence of biotic and abiotic factors. The rate of oxygen consumption of the whole animal as well as its tissues reflects the metabolic rate, which can be taken as index of the metabolic status of the animal. Therefore, measurement of $\mathrm{O}_{2}$ consumption can be constructed as a bio-detector of pollution of different types including heavy metal pollution, the toxic nature causes stress to the animal. The rate of $\mathrm{O}_{2}$ consumption is taken as a parameter to assess the toxic impact of $\mathrm{Cd}$ and will provide useful information on the energy metabolism. Since, the present work concerns the metabolic status of the rats under $\mathrm{Cd}$-toxicity, study of whole animal and tissue respiratory potentials is essential to determine the metabolic status. The results obtained in the present clearly demonstrates that the Cd-toxicity induces hypoxic stress condition in rats and are reflected through a decreased $\mathrm{O}_{2}$ consumption at the tissue level i.e. liver and kidney tissue. In agreement with Total respiration, the unit metabolism also showed a significant decrease under Cd-intoxication. This indicates that there was a shift in the metabolic emphasis from aerobiosis to anerobiosis as a sort of metabolic compensation to overcome the Cd-toxicity.

In the present study, an attempt was made to evaluate $\mathrm{Cd}$ toxic effects in rats, furthermore we are very much interested to know the amelioration of Vitamin C, Zinc, NAC coadministration with Cd, individually and also in combinations. Cadmium has been recognized as one of the most toxic environmental and industrial pollutants and has been reported to induce oxidative damage. Due to the disruption of prooxidant-antioxidant balance at cellular level or tissues, induces severe oxidative stress. Cd induced oxidative damage was previously observed in rats treated with this metal $[6,1]$. The seriousness of intoxication be governed by on the route, dose and duration of exposure to $\mathrm{Cd}$ [2]. In cells, Cd mainly accumulates in the cytosol (70\%), followed by the nucleus (15\%) and small amounts are found to be in the mitochondria and endoplasmic reticulum [23]. However, administration of Cd with chemical or mineral or vitamin supplements was previously shown to diminish the toxic effects of Cd [6]. In the present study, SOD, CAT and GPx activities were found to be significantly decreased, and also decreases the activity of redox cycling antioxidant enzymes and rises lipid peroxidation in liver and kidney tissues of rats. This effect has been correlated with oxidative stress. The activities of SOD and CAT were decreased in Cd-treated rats and increased in rats administered with Vitamin-C, Zinc, NAC either individually or in combinations. The decreased activities of SOD and CAT may be due to concomitant increase in the generation of free radicals in the 
tissues of rats, consequent upon $\mathrm{Cd}$-administration. The interaction between $\mathrm{Cd}$ and essential trace elements may be one of the reasons for the decrease in antioxidant enzymes in the tissues of rats including liver and kidney, because Cd can occupy the Zinc site in $\mathrm{Cu} / \mathrm{Zn}-\mathrm{SOD}$ and creates inactive forms of the enzyme (Cu/Cd-SOD) [24]. However, the activity of Cu/Zn-SOD was increased in rats supplemented with Vitamin C, Zinc, NAC, this may occur because the supplements protect against the cytotoxicity of Cd, permitting the maintenance of normal cellular redox balance by blocking the free radical generation. The decrease in the liver SOD activity is possibly an expected result that occurs due to lipid peroxyl radicals and from an inactivation of their destruction products. There is a tendency that, increased MDA levels and reduced SOD activities would favorably support thus hypothesis. The increase in protein oxidation levels and lipid peroxides is studied in many individuals with a known liver disease induced by exposure to hepato-toxic agents [25]. Significant decreases in the activity of GPx in Cd-treated rats may be due to the enhancement of peroxidative damage to polyunsaturated fatty acids, which results in the highest levels of lipid peroxidation in different tissues or to the accumulation of ROS with subsequent development of tissue injury. This suggests that free radicals could be involved in the damage caused by long-term exposure to $\mathrm{Cd}$ [26]. Cd may reduce the efficiency of GPx only in part via its direct inhibitory effect on the enzyme, which indirectly leads to a shortage of the reduced glutathione and NADPH required for its activity [27]. However, changes in GPx activity caused by Cd toxicity, was ameliorated by the supplementation with co-administration of Vitamin C, Zinc, NAC enhances the antioxidative defense system, thereby providing protection against Cd-toxicity [28]. Cd roots significant increases in LPx concentrations in rat tissues and causes lipid peroxidation in numerous tissues [29]. Which suggests that CD may persuade oxidative stress by producing hydroxyl radicals, superoxide ions, nitric oxide and $\mathrm{H}_{2} \mathrm{O}_{2}$ [5]. The activity of LPx was significantly decreased in rats co-supplemented with Vitamin C, Zinc and NAC individually and also in combinations due to the effects of the antioxidant defense system, which protects cells from Cd-induced toxicity. Thus, supplements have roles in maintaining the LPx level and in protecting the integrity and function of tissues [8,30]. It is acknowledged that hepatic GSH acts as an electrophile, radical scavenger and redox partner. GSH may also serve as a co-factor for several drug- metabolizing enzymes i.e. GSTs, which it is consumed or for antioxidant enzymes i.e. GPx, where it serves as a redox partner [31]. During oxidation, GSH forms a dimer, glutathione disulfide (GSSG), which, inturn can be reduced by the enzyme Glutathione reductase at the expense of NADPH [31]. The reduction in activities of antioxidant enzymes in our study is in line with several earlier studies reported the same disorders [1,22]. Organisms may have an endogenous protective antioxidant defend system against the damages of free oxygen radicals. SOD, CAT and GPx are enzymatic antioxidants that catalyze detoxification reactions of toxic oxygen metabolites. The failure of the primary antioxidant system to act against free radicals generated may reflect the inability of liver mitochondria and microsomes to eliminate $\mathrm{H}_{2} \mathrm{O}_{2}$ produced after exposure to Cd. This may also be due to enzyme inactivation caused by excess ROS production in mitochondria and microsomes [32]. All these findings are confirmed through histopathological studies (unpublished data), showing congestions of hepatoportal blood vessels, inflammatory infiltrations, degeneration of hepatocytes with necrotic foci scattered throughout the liver. Although several experimental studies have shown that $\mathrm{Cd}$ is relatively rapidly metabolized and excreted in mammalian models including rats $[1,33]$. It was reported that an accumulation of $\mathrm{Cd}$ in several target organs including liver, gonads, brain etc. causing damage at cellular level leading to disturbances in several metabolic and biochemical pathways following exposure to different doses of $\mathrm{Cd}[1,33]$

From the results obtained that, Cd-toxicity induces the damage that occurred in liver and kidney tissues as observed through pathological studies. Both liver and kidney were considered as important organs concerning the conduct of metabolic events including detoxification, storage and excretion of xenobiotics and their metabolites. Due to physiological and biochemical functions assigned to the liver and kidney clearly suggests that they are especially vulnerable to damage. In the present study an attempt was also made to assess the impact of Cd-toxicity and followed by its amelioration with co- administration of Vitamin C, Zinc, NAC both individually and also in combinations in rats. Serum enzymes including AST, ALT, LDH and biomolecules like Albumin, Urea and Creatinine were mainly considered as biomarkers for the evaluation of hepatic damage under Cd toxicity. In the present study, Cdtoxicity causes a significant elevation in the activity levels of several serum enzymes like AST, ALT and LDH were recorded. In contrast, ALP activity showed a significant decrease in the activity compared to control group. In addition to the above, increased levels of hepatic serum markers also suggests an extensive damage to the liver tissue during Cd-toxicity. LPO is one of the main manifestations of oxidative damage, which always plays an important role in the toxicity of several xenobiotics [33]. Moreover it has been confirmed through the data obtained under Cd-toxicity causes a significant increase in lipid peroxidation concentrations in liver tissue of rats, since it causes LPO in numerous tissue both in vivo and invitro [34]. Cd-toxicity may likely to induce oxidative stress by the production of hydroxyl radicals, superoxide anions, nitric oxide and $\mathrm{H}_{2} \mathrm{O}_{2}$ [33]. Moreover Cd-treatment also causes both structural and functional damage to the cell membrane, significantly increasing the permeability, thereby results in the leakage of hepatic enzymes into blood. Furthermore, the liver damage was also confirmed during Cd-toxicity with a significant increase in the levels of plasma components including albumin, bilirubin etc. Therefore, the increased activities of ALT \&AST in plasma clearly attributes to 
the leakage of these enzymes from the liver cytosol into the bloodstream. Earlier reports also suggested that lysosomal instability caused by Cd-toxicity resulted in the leakage of hepatic enzymes including ALT, AST and ALP into the blood stream [35]. In consonance with the above observations, there was a significant increased activity levels were recorded with AST \& ALT, more over changes with ALT activities may also be attributed to the significant damage occurred during Cd-toxicity. Moreover, the changes in ALP activities also be contributed to cholestasis and acute hepatocellular necrosis. Earlier reports also available that Cd-toxicity causes a significant elevation in liver enzymes including SGOT, SGPT and ALP, clearly suggesting the liver dysfunction. The kidneys are responsible for the elimination of unmodified drugs and metabolites. Additionally, these organs are also capable to realize diffused biotransformation reactions. The present studies clearly demonstrate that nephrotoxicity induced by chemical agents are use of the consequences of accumulation of certain metabolites in kidneys. Kidney damage is one of the most prominent reasons of death due to Cd- toxicity. Earlier reports are also available about Cd-effects on kidney. Moreover effects of Cd-toxicity in liver and kidney, and pathology related to both nephrotoxicity and hepatotoxicity needs further in depth investigation during Cd- toxicity, to answer several queries. The tissues have higher affinity against $\mathrm{Cd}$ molecules, and as they contain predominantly, cytochrome P450. Several authors reported that Cd-toxicity disrupts the structural and functional organization of both liver and kidney [1]. LPO is a lipid peroxidation marker. The majority of researcher's quantify the level of malondialdehyde (MDA) as the indicator of oxidative damage to lipid compounds. However, in order to accurately assess this process, LPO levels were assayed. The increase of LPO level in the liver and kidney tissues of $\mathrm{Cd}$ - exposure as compared to the control group, indicates not only the intensification of lipid peroxidation but also the significant rise of oxidative stress. An important indicator of oxidative cellular damage is also the $\mathrm{H}_{2} \mathrm{O}_{2}$ which is a natural product of cellular metabolism. Due to its strong oxidation properties, that compound is highly reactive and also toxic to the cell. Under normal physiological conditions, $\mathrm{H}_{2} \mathrm{O}_{2}$ is deactivated by CAT and GPx. Those enzymes prevent excessive accumulation of $\mathrm{H}_{2} \mathrm{O}_{2}$ in cells, thus protecting the organism against the destructive effect of that compound on proteins, lipids or nucleic acids. The available study outcomes suggests that antioxidants such as vitamins, polyphenyls, metals may protect the organism exposed to $\mathrm{Cd}$ toxicity, against oxidative stress. Increased serum, urea and creatinine levels in the $\mathrm{Cd}$ - treated rat group may be attributed to an oxidative imbalance in the liver and kidney, leading to elevated urea and creatinine in the blood and correlate with the earlier reports [36], where the urea level was elevated as a result of Cd-toxicity. The significantly elevated level of creatinine during Cd-toxicity may be attributed to the oxidative damage to the tissues like liver and kidney. Deterioration of the tissue architecture during Cd toxicity, would permit creatinine release into a blood and agree with earlier reports, where Cd administration leads to an elevated level of creatinine [36]. Tissue damage was also linked to the defect in infiltration. Earlier reports also available that rise in creatinine levels is an indication of renal-tubular damage due to Cd-induced nephrotoxicity [36,2].

In the present study an attempt was also made to monitor the rate of amelioration of Cd-toxicity by co-administration of Vitamin C, Zinc and NAC in rat tissues like liver and kidney. A very effective therapeutic way commonly used for heavy metal poisoning in chelation therapy, which promotes the excretion of metals. Natural antioxidant compounds such as vitamin C \& E which, besides possessing activity, are also good chelators used in metal toxicity management. Vitamin C (Ascorbic acid) is an important dietary antioxidant, it significantly decreases the adverse effects of reactive species such as reactive oxygen and nitrogen species that can cause oxidative damage to macromolecules such as lipids, proteins, DNA, which are implicated in chronic diseases including cardiovascular disease, stroke, cancer, neurodegenerative diseases and cataractogenesis. Ascorbic acid is a potent water soluble antioxidant capable of scavenging or neytralizing an array of ROS including hydroxyl, alkolyl, peroxyl, superoxide anion, hydroperoxyl radicals and reactive nitrogen radicals such as nitrogen dioxide, nitroxide, peroxynitrile at very low concentrations $[37,38]$. Vitamin $\mathrm{C}$ is an essential co-factor for many enzymes involved in diverse metabolic pathways. In the present study co-administration of vitamin $\mathrm{C}$, significantly ameliorated the Cd-toxicity in rat tissues, which were monitored through activity levels of antioxidant enzymes in tissues and also serum. Previously reports are also available that vitamin C significantly protected the Cd-toxicity i.e. activity levels of ALT, AST, SOD, CAT, SGOT, SGPT were significantly altered in Cd-induced toxicity in rats and rabbits $[39,40,38]$.

It is well known that endogenous antioxidants may play an imperative role in antioxidant defense against oxidative impairment, possibly by protecting the biological cell functions [41]. Among the range of antioxidants, Zinc play an important role in the regulation of antioxidant system [42,36]. Zn interacts with heavy metals such as $\mathrm{Cd}$ in metabolism and toxicity [43]. Accordingly, Zn neutralizes the toxic effect of $\mathrm{Cd}$ in many tissues, especially it prevents kidney damages. The results obtained in the present study also clearly reveal the protective nature of Cd-toxicity in rat tissues. Many studies have indicated that $\mathrm{Zn}$ has protective effects on Cdinduced changes on the oxidative enzymes such as SOD, CAT, GPx etc. Moreover competitive absorption of Cd and $\mathrm{Zn}$ can influence $\mathrm{Cd}$ susceptibility. Enhanced daily $\mathrm{Zn}$ intake has been suggested to protect the organism against Cd-accumulation and subsequently toxicity. 
In one of the experimental group, NAC was co-administered with $\mathrm{Cd}$ to monitor the amelioration potential in rat tissues. NAC administration was effective enough in reducing oxidative damage in both the tissues like liver and kidney of rats, thereby increasing the antioxidant status most efficiently.

\section{Conclusions}

In conclusion, the present study demonstrated that the cadmium is a toxic element that causes damages at liver and kidney tissues of rats. Vitamin C, Zinc and NAC have been proven to have protective roles against Cd-toxic effects. The results obtained in the present study pertaining to Basal Metabolic rate, Tissue Respiratory potentials, antioxidant enzyme activity levels and serum biomarker enzymes and antioxidant product quantities clearly demonstrates that all the antioxidant agents selected in the present study Vitamin C, Zinc and NAC had nearly the same protective effects against Cd-toxicity at tissue level. The co-administration of Vitamin C, Zinc and NAC with Cd, suggests that beneficial effects in the amelioration of Cd toxicity. Further studies are necessary to explain other possible protective effects of the above selected agents in subjects exposed to $\mathrm{Cd}$ and to establish the exact pathways or reactions at cellular level.

\section{Declarations}

\section{Acknowledgements}

The authors would like to acknowledge the Department of Zoology of the Sri Venkateswara University and Technology, for allowing us to use their laboratories for our research work.

\section{Author contributions}

PVR was responsible for the article Collected, analyzed and interpreted the data. MR was responsible for the article interpreted the data. MSR was responsible for the article Supervised data collection and conceptualization and design of the article, experimental works, data analysis, literature search, writing-original draft preparation, drafting of the article, revision of the text, and final approval of the article. The final manuscript has been approved by all authors.

\section{Funding}

This research acquired no particular provide from any funding agency in the public, commercial or non-income sectors.

\section{Data availability}

Data generated during this research work can be available upon request.

\section{Conflict of interest}

The authors declare that they no regard competing financial hobbies or private relationships that might have seemed to influence the work reported in this paper.

\section{Ethical approval}

The study was approved by the Institutional Animal Ethics Committee has approved the Experimental protocols and animal use (Resolution No. 60b/2012/(i)/a/CPCSEA/IAEC/SVU/ MSR-RS dt. 08.07.2012), Sri Venkateswara University, Tirupati, Andhra Pradesh, India.

\section{Consent to participate}




\section{Consent for publication}

All authors have agreed with the content and all have given explicit consent to publish.

\section{References}

1. Sandra C, Das Hamda A, Al-Naemi (2019) Cadmium toxicity: Oxidative stress, inflammation and tissue injury. Occupational Diseases and Environmental Medicine 7: 144-163

2. Mohammad NS, Tangpong J, Rahman MM (2018) Toxicodynamics of Lead, Cadmium, Mercury and Arsenic induced kidney toxicity and treatment strategy: A mini review. Toxicology Reports 5: 704-713

3. Bull S (2010) Cadmium. Toxicological overview. Health Protection Agency 3: 3-15

4. IPCS (2010) International Programme on Chemical Safety. Cadmium. Poisons Information Monograph. PIM 089. http://www.inchem.org/documents/pims/chemical/cadmium. htm

5. Genchi G, Maria SS, Lauria G et al (2020) The Effects of Cadmium Toxicity. Int J Environmental Research and Public Health 17: 3782-3806

6. Jacopo JVB, Fiorillo C, Carrino D, Paternostro F, Taddei N, Gulisano M, Pacini A, Becatti M (2020) Cadmium-induced stress: Focus on the central nervous system. Antioxidants 9: 492-513

7. Milena A, Aleksandra BD, Evica A et al (2019) Toxic effect of Acute Cadmium and Lead exposure in Rat blood, liver and kidney. Int J of Environmental Research and Public Health 16: 274-295

8. Brzoska MM, Borowska S, Tomezyk M (2016) Antioxidants as a potential preventive and therapeutic strategy for Cadmium. Current Drug Targets 17: 1350-1384

9. Amel SM, Loukidi B, Reda B et al (2020) Effects of vitamin C and E against oxidative stress: Is Antioxidant supplementation Efficient? Current Neutraceuticals 1: 33-41

10. Elias A, Deo O (2013) Hepatoprotective effect of Vitamin C (Ascorbic acid). Pharmacology \& Pharmacy 4: 84-92

11. Umbreit WW, Burris RH, Stauffer JF (1964) Manometric techniques. Burgess

12. Lowry OH, Rosenbrough NJ, Farr AL and Randall RJ (1951) Protein measurement with the Folin phenol reagent. J Biol Chem 193: $265-275$

13. Patton CJ, Crouch SR (1977) Spectrophotometric and kinetics investigation of the Berthelot reaction for the determination of ammonia. Anal Chem 49: 464-469

14. Faulkner WR, King JW (1976) Renal function. In; Tietz N. Eds. Fundamentals of Clinical Chemistry. Philadelphia. USA. WB. Samders co 975-1014

15. Kornberg A (1974) Methods of Enzymatic Analysis. Bergmeyer HO. Newyork. USA. Academic Press

16. Reitman S, Frankel S (1957) A colorimetric method for determination of serum ALT and AST. Am J Clin Pathol 28: 56-63

17. Ohkawa H, Ohishi N, Yagi K (1979) Assay for lipid peroxides in animal tissues by thiobarbituric acid reaction. Analytical Biochemistry 95: 351-358

18. Misra HP, Fridovich I (1972) The role of superoxide anion in the autooxidation of epinephrine and a simple assay for superoxide dismutase. J Biol Chem 247(10): 3170-3175

19. Aebi H (1984) Catalase in vitro. Methods in Enzymology 105: 121-126

20. Wendel Abrecht (1981) Glutathione Peroxidase. Methods in Enzymology 77: 325-333

21. Moron MS, Depierre JW, Mannervik B (1979) Levels of glutathione, glutathione reductase and glutathione S-transferase activities in rat lung and liver. Biochem Biophys Acta 582(1):67-78

22. Renuka M, Aparna $Y$, venkataramanaiah $P$ et al (2021) Vitamin $C, E$ and Zinc ameliorates Cadmium toxicity induced biochemical changes in male albino rats. Toxicology \& Forensic Medicine 6 (1): 13-19

23. Casalino E, Sblano C, Landriscina C (1997) Enzyme activity alteration by Cadmium administration to rat: The possibility of iron involvement in lipid peroxidation. Arch Biochem Biophys 346: 171-179. 1997 
24. Bauer R, Demeter I, Hasemann V et al (1980) Structural properties of the Zinc site in the cup, n-superoxide dismutase: Perturbed angular correlation of gamma ray spectroscopy on the $\mathrm{Cu}-111 \mathrm{Cd}$-Superoxide Dismutage derivative. Biochem Biophys Res Commum 94:1296-1302

25. Harikara, Aydm C, Vahit K et al (2008) Effects of selenium with Vitamin E and melatonin on cadmium- induced oxidative damage in rat liver and kidneys. Biol Trace Elem Res 125;236-244

26. Patra RC, Swarup D, Senapati SK (1999) Effects of cadmium on lipid peroxides and superoxide dismutase in hepatic, renal and testicular tissue of rats. Vet Hum Toxicol 41: 65-67

27. Shukla GS, Chandra SV (1989) Cadmium toxicity and bioantioxidants: status of Vitamin E and ascorbic acid of selected organs in rat. J Appl Toxicol 9: 119-122

28. Shaikh ZA, Vu TT, Zaman K (1999) Oxidative stress as a mechanism of chronic cadmium induced hepatotoxicity and renal toxicity and protection by antioxidants. Toxicol Appl Pharmacol 154: 256-263

29. Kostić MM, Ognjanović B, Dimitrijević S et al (1993) Cadmium-induced changes of antioxidant and metabolic status in red blood cells of rats: in vivo effects. Eur J Haematol 51: 86-92

30. Zorica PB, Danijela DC, Zivorad M et al (2008) Zinc or Magnesium supplementation modulates cadmium intoxication in blood, kidney, spleen and bone of rabbits. Biol Trace Elem Res 124: 110-117

31. Mates JM (2000) Effects of antioxidant enzymes in the molecular control of reactive oxygen species toxicology. Toxicology 153 : 83-104

32. Giuseppe C, Elisa F, Claudio L et al (2009) Cadmium and mitochondria. Mitochondrion 9: 377-384

33. Milena Andjelkovic, Aleksandra Buha Djordjevic, Evica Antonijevic et al (2019) Toxic Effect of Acute Cadmium and Lead Exposure in Rat Blood, Liver, and Kidney. Int J Environ Res Public Health 16: 274

34. Koyu A, Gokcimen A, Ozguner F et al (2006) Evaluation of the effects of cadmium on rat liver. Molecular and Cellular Biochemistry 284: 81-85

35. Slencu BG, Ciobanu C, Solcan C et al (2014) Effect of Selenium Supplementation on Serum amylase, Lactate dehydrogenase and Alkaline phosphatase activities in rats exposed to Cadmium or Lead. Cercetari Agronomice in Moldova 4(160): 113-121

36. Nasim B, Moshtaghie AA, Nayeri H et al (2016) Protective role of Zinc and Magnesium against Cadmium nephrotoxicity in male wistar rats. Biol Trace Elem Res 174(1): 112-120

37. Carr AC, Frei B (1999) Does vitamin C act as a pro-oxidant under physiological conditions? The Federation of American Societies for Experimental Biology. FASEB J 13: 1007-1024

38. Tarasub N, Junseecha T, Tarasub C et al (2012) Protective effects of Curcumin, Vitamin C, or their Combination on Cadmiuminduced hepatotoxicity. Journal of Basic and Clinical Pharmacy 3(2):273-281

39. Yousef MI, Salem MH, Kamel KI et al (2003) Influence of ascorbic acid supplementation on the haematological and clinical biochemistry parameters of male rabbits exposed to aflatoxin B1. J Environ Sci Health 38(2):193-209

40. Yousef MI (2004) Aluminium-induced changes in hemato-biochemical parameters, lipid peroxidation and enzyme activities of male rabbits: protective role of ascorbic acid. Toxicology 199(1):47-57

41. Gunther MR (2004) Probing the free radicals formed in the metmyoglobin-hydrogen peroxide reaction. Free Radic Biol Med 36: $1345-1354$

42. Pradeepkiran JA, Siva Prasad B, Rajesh AV et al (2016) Calcium, Zinc and Vitamin E ameliorate Cadmium-induced renal oxidative damage in albino rats. Toxicology Reports 3: 591-597

43. Arif TJ, udsser Azam, Siddiqui K et al (2015) Heavy metals and human health: Mechanistic insight into toxicity and counter defence system of Antioxidants. Int J Mol Sciences 16: 29592-29630

\section{Tables}

\section{Table 1}

Body and relative organ weight in control and experimental rats. 


\begin{tabular}{|c|c|c|c|c|c|c|c|c|c|}
\hline Parameters & Control & $\begin{array}{l}\mathrm{Cd} \\
\text { treated }\end{array}$ & Vit-C & Zinc & NAC & $C+Z$ inc & $\mathrm{C}+\mathrm{NAC}$ & $\begin{array}{l}\text { Zinc+ } \\
\text { NAC }\end{array}$ & $\begin{array}{l}\text { C+Zinc } \\
+ \text { NAC }\end{array}$ \\
\hline \multirow[t]{2}{*}{ IBW } & 225.19 & 226.12 & 225.18 & 224.19 & 225.49 & 225.42 & 226.74 & 225.48 & 224.14 \\
\hline & \pm 10.12 & \pm 10.14 & \pm 9.79 & \pm 10.17 & \pm 10.15 & \pm 10.19 & \pm 10.45 & \pm 10.12 & \pm 10.15 \\
\hline \multirow[t]{2}{*}{ FBW } & $268.15^{a}$ & $122.35^{\mathrm{b}}$ & $238.14^{b . c}$ & $237.19^{b . c}$ & $238.32^{\text {b.c }}$ & $238.05^{b . c}$ & $237.78^{b . c}$ & $238.04^{\text {b.c }}$ & $238.05^{\text {b.c }}$ \\
\hline & \pm 10.17 & \pm 10.22 & \pm 10.12 & \pm 10.03 & \pm 10.05 & \pm 10.14 & \pm 9.89 & \pm 9.88 & \pm 10.13 \\
\hline BWG/L & $+19.07^{b}$ & $-45.89^{a}$ & $+5.76^{b}$ & $+5.79^{b}$ & $+5.69^{b}$ & $+5.60^{b}$ & $+4.87^{b}$ & $+5.57^{b}$ & $+5.60^{b}$ \\
\hline \multirow[t]{3}{*}{ LW } & $10.84^{a}$ & $4.82^{b, c}$ & $8.12^{b, c}$ & $8.24^{b, c}$ & $8.25^{b, c}$ & $8.18^{b, c}$ & $8.25^{b, c}$ & $8.05^{b, c}$ & $8.19^{b, c}$ \\
\hline & \pm 0.63 & \pm 0.25 & \pm 0.45 & \pm 0.38 & \pm 0.39 & \pm 0.42 & \pm 0.41 & \pm 0.38 & \pm 0.35 \\
\hline & PDC & -55.54 & -25.09 & -23.99 & -23.89 & -32.52 & -23.89 & -27.74 & -24.45 \\
\hline \multirow[t]{2}{*}{ HIS-L } & $4.04^{a}$ & $3.94^{\mathrm{a}}$ & $3.41^{\mathrm{b}}$ & $3.48^{b}$ & $3.46^{b}$ & $3.44^{\mathrm{b}}$ & $3.47^{b}$ & $3.38^{b}$ & $3.44^{\mathrm{b}}$ \\
\hline & PDC & -2.48 & -15.59 & -13.86 & -14.36 & -14.85 & -14.11 & -16.34 & -14.85 \\
\hline \multirow[t]{3}{*}{$\mathrm{KW}$} & $2.93^{a}$ & $1.23^{b}$ & $2.08^{b, c}$ & $2.12^{b, c}$ & $2.11^{b, c}$ & $2.14^{b, c}$ & $2.14^{b, c}$ & $2.12^{b, c}$ & $2.11^{b, c}$ \\
\hline & \pm 0.28 & \pm 0.14 & \pm 0.15 & \pm 0.17 & \pm 0.14 & \pm 0.17 & \pm 0.18 & \pm 0.15 & \pm 0.15 \\
\hline & PDC & -44.37 & -29.01 & -27.65 & -27.99 & -29.96 & -26.96 & -27.65 & -27.99 \\
\hline \multirow[t]{2}{*}{ HIS-K } & $1.09^{a}$ & $1.01^{\mathrm{a}}$ & $0.87^{b}$ & $0.89^{b}$ & $0.89^{b}$ & $0.90^{\mathrm{b}}$ & $0.90^{\mathrm{b}}$ & $0.89^{b}$ & $0.89^{b}$ \\
\hline & PDC & -7.34 & -20.18 & -18.35 & -18.35 & -17.43 & -17.43 & -18.35 & -18.35 \\
\hline $\begin{array}{l}\text { Values are ex } \\
\text { differ signific } \\
\text { Weight (g). B } \\
\text { Weight (g). }\end{array}$ & $\begin{array}{l}\text { ssed as } \\
\text { ly at (P. } \\
/ \mathrm{L}(\%) \text { : } \\
\text { C: Histos }\end{array}$ & $\begin{array}{l}\text { an } \pm \text { SD } \\
(\text { OMR } \\
\text { Weigh } \\
\text { atic Ind }\end{array}$ & $\begin{array}{l}\text { individua } \\
\text { C: Percep } \\
\text { LLoss g i } \\
\text { idney. }\end{array}$ & $\begin{array}{l}\text { servatic } \\
\text { eviation } \\
\text { days. }\end{array}$ & $\begin{array}{l}\text { a,b,c Valu } \\
\text { Control. } \\
\text { iver Weig }\end{array}$ & $\begin{array}{l}\text { Ire not sh } \\
\text { IBW } \\
\text { g). HIS-L }\end{array}$ & $\begin{array}{l}\text { Ig a com } \\
\text { ial Body } \\
\text { tosomat }\end{array}$ & $\begin{array}{l}\text { superscri } \\
\text { ht (g). FB } \\
\text { ex of live }\end{array}$ & $\begin{array}{l}\text { tter }(a, b, c) \\
\text { Tinal Body } \\
\text { N: Kidney }\end{array}$ \\
\hline
\end{tabular}

Table 2

Basal Metabolic Rate (BMR) and Tissue Respiration in Selected tissue in Control and Experimental rats. 


\begin{tabular}{|c|c|c|c|c|c|c|c|c|c|}
\hline Parameters & Control & $\begin{array}{l}\mathrm{Cd} \\
\text { treated }\end{array}$ & Vit-C & Zinc & NAC & $C+Z$ inc & $\mathrm{C}+\mathrm{NAC}$ & $\begin{array}{l}\text { Zinc+ } \\
\text { NAC }\end{array}$ & $\begin{array}{l}\text { C+Zinc } \\
+\mathrm{NAC}\end{array}$ \\
\hline \multirow{3}{*}{$\begin{array}{l}\text { Basal } \\
\text { Metabolic } \\
\text { Rate (BMR) }\end{array}$} & $4.23^{a}$ & $2.72^{\mathrm{b}}$ & $3.41^{b, c}$ & $3.54^{b, c}$ & $3.53^{b, c}$ & $3.54^{b, c}$ & $3.48^{b, c}$ & $3.53^{b, c}$ & $3.54^{b, c}$ \\
\hline & \pm 0.24 & \pm 0.13 & \pm 0.13 & \pm 0.15 & \pm 0.16 & \pm 0.13 & \pm 0.15 & \pm 0.14 & \pm 0.15 \\
\hline & PDC & -35.70 & -19.39 & -16.31 & -16.55 & -16.31 & -17.73 & -16.55 & -16.31 \\
\hline \multirow{3}{*}{$\begin{array}{l}\text { Tissue } \\
\text { Respiration } \\
\text { (TR), Liver }\end{array}$} & $1033.46^{a}$ & $642.14^{b}$ & $842.18^{b, c}$ & $85214^{b, c}$ & $860.15^{b, c}$ & $862.19^{b, c}$ & $863.14^{b, c}$ & $861.15^{b, c}$ & $867.18^{b, c}$ \\
\hline & \pm 24.77 & \pm 18.15 & \pm 16.19 & \pm 17.15 & \pm 17.19 & \pm 17.14 & \pm 16.75 & \pm 17.13 & \pm 14.19 \\
\hline & PDC & -37.87 & -18.51 & -17.55 & -16.77 & -16.57 & -16.48 & -16.67 & -16.09 \\
\hline \multirow{3}{*}{$\begin{array}{l}\text { Tissue } \\
\text { Respiration } \\
\text { (TR), Kidney }\end{array}$} & $858.77^{a}$ & $508.42^{b}$ & $660.18^{b, c}$ & $672.13^{b, c}$ & $673.13^{b, c}$ & $671.72^{b, c}$ & $675.70^{b, c}$ & $673.49^{b, c}$ & $677.43^{b, c}$ \\
\hline & \pm 12.85 & \pm 12.12 & \pm 12.19 & \pm 15.14 & \pm 11.14 & \pm 13.49 & \pm 14.19 & \pm 13.45 & \pm 14.46 \\
\hline & PDC & -40.80 & -23.12 & -21.73 & -21.61 & -21.78 & -21.32 & -21.58 & -21.12 \\
\hline
\end{tabular}

\section{Table 3}

Biochemical Parameters in Serum of rats under different Experimental treatments. 


\begin{tabular}{|c|c|c|c|c|c|c|c|c|c|}
\hline Parameters & Control & $\begin{array}{l}\mathrm{Cd} \\
\text { treated }\end{array}$ & Vit-C & Zinc & NAC & $C+Z$ inc & $\mathrm{C}+\mathrm{NAC}$ & $\begin{array}{l}\text { Zinc+ } \\
\text { NAC }\end{array}$ & $\mathrm{C}+\mathrm{Zinc}+\mathrm{NAC}$ \\
\hline \multirow{3}{*}{$\begin{array}{l}\text { Total } \\
\text { Protein } \\
(\mathrm{mg} / \mathrm{ml})\end{array}$} & $15.38^{a}$ & $33.49^{b}$ & $20.31^{b, c}$ & $25.34^{b, c}$ & $26.77^{b, c}$ & $26.82^{b, c}$ & $22.14^{b, c}$ & $20.74^{b, c}$ & $20.18^{b, c}$ \\
\hline & \pm 1.29 & \pm 2.28 & \pm 1.72 & \pm 1.83 & \pm 1.49 & \pm 1.34 & \pm 1.13 & \pm 1.14 & \pm 1.12 \\
\hline & PDC & +117.75 & +32.05 & +64.48 & +74.06 & +74.97 & +43.95 & +34.85 & +31.21 \\
\hline \multirow{3}{*}{$\begin{array}{l}\text { Albumin } \\
\text { (mg/ml) }\end{array}$} & $2.38^{a}$ & $2.89^{b}$ & $3.04^{b, c}$ & $3.08^{b, c}$ & $3.12^{b, c}$ & $2.94^{b, c}$ & $2.95^{b, c}$ & $2.99 \mathrm{~b}, \mathrm{c}$ & $3.02^{b, c}$ \\
\hline & \pm 0.22 & \pm 0.23 & \pm 0.23 & \pm 0.24 & \pm 0.25 & \pm 0.22 & \pm 0.22 & \pm 0.24 & \pm 0.22 \\
\hline & PDC & +21.43 & +27.73 & +29.41 & +31.09 & +23.53 & +23.95 & +25.63 & +26.89 \\
\hline \multirow{2}{*}{$\begin{array}{l}\text { Urea } \\
(\mathrm{mg} / \mathrm{dl})\end{array}$} & $34.14^{a}$ & $41.19^{b}$ & $38.77^{b, c}$ & $39.42^{b, c}$ & $38.45^{b, c}$ & $39.25^{b, c}$ & $37.77^{b, c}$ & 38.41 & $36.77^{b, c}$ \\
\hline & \pm 2.12 & \pm 2.74 & \pm 2.49 & \pm 2.49 & \pm 2.44 & \pm 2.38 & \pm 2.74 & \pm 2.49 & \pm 1.42 \\
\hline \multirow{3}{*}{$\begin{array}{l}\text { Creatinine } \\
(\mathrm{mg} / \mathrm{dl})\end{array}$} & $0.65^{a}$ & $0.83^{b}$ & $0.82^{b, c}$ & $0.79^{b, c}$ & $0.78^{b, c}$ & $0.77^{b, c}$ & $0.74^{b, c}$ & $0.73^{b, c}$ & $0.74^{b, c}$ \\
\hline & \pm 0.04 & \pm 0.08 & \pm 0.07 & \pm 0.08 & \pm 0.07 & \pm 0.07 & \pm 0.08 & \pm 0.08 & \pm 0.07 \\
\hline & PDC & +20.62 & +13.56 & +21.54 & +20.00 & +18.46 & +13.85 & +12.31 & +13.85 \\
\hline \multirow{3}{*}{$\begin{array}{l}\text { LDH } \\
\text { ( } \mu \text { moles of } \\
\text { formazon } \\
\text { formed/ } \mathrm{mg} \\
\text { protein } / \mathrm{hr}\end{array}$} & $18.14^{a}$ & $39.79^{b}$ & $35.14^{b, c}$ & $34.72^{b, c}$ & $27.45^{b, c}$ & $25.79^{b, c}$ & $24.74^{b, c}$ & $21.74^{b, c}$ & $25.78^{b, c}$ \\
\hline & \pm 0.25 & \pm 0.34 & \pm 0.29 & \pm 0.27 & \pm 0.25 & \pm 0.34 & \pm 0.31 & \pm 0.31 & \pm 0.29 \\
\hline & PDC & +119.35 & +93.72 & +91.40 & +51.32 & +42.18 & +36.38 & +19.85 & +42.12 \\
\hline \multirow{3}{*}{$\begin{array}{l}\text { AST } \\
(\mathrm{U} / \mathrm{L})\end{array}$} & $53.14^{\mathrm{a}}$ & $459.78^{b}$ & $254.72^{b, c}$ & $277.45^{b, c}$ & $245.42^{b, c}$ & $205.74^{b, c}$ & $214.12^{b, c}$ & $205.74^{b, c}$ & $201.71^{b, c}$ \\
\hline & \pm 2.77 & \pm 25.85 & \pm 15.75 & \pm 18.74 & \pm 19.45 & \pm 18.42 & \pm 20.14 & \pm 18.85 & \pm 18.45 \\
\hline & PDC & +765 & +379 & +422 & +361 & +287 & +303 & +287 & +279 \\
\hline \multirow{3}{*}{$\begin{array}{l}\text { ALT } \\
(\mathrm{U} / \mathrm{L})\end{array}$} & $39.77^{a}$ & $365.72^{b}$ & $204.14^{b, c}$ & $201.45^{b, c}$ & $194.15^{b, c}$ & $190.45^{b, c}$ & $185.45^{b, c}$ & $168.74^{b, c}$ & $158.74^{b, c}$ \\
\hline & \pm 2.04 & \pm 20.14 & \pm 15.05 & \pm 19.41 & \pm 19.42 & \pm 20.42 & \pm 20.14 & \pm 14.55 & \pm 12.85 \\
\hline & PDC & +196 & +413 & +407 & +388 & +379 & +366 & +324 & +299 \\
\hline \multirow[t]{3}{*}{ ALP } & $108.12^{a}$ & $38.19^{b}$ & $62.13^{b, c}$ & $68.34^{b, c}$ & $70.13^{b, c}$ & $73.14^{b, c}$ & $75.18^{b, c}$ & $78.11^{b, c}$ & $80.13^{b, c}$ \\
\hline & \pm 8.14 & \pm 2.15 & \pm 3.75 & \pm 3.49 & \pm 3.74 & \pm 4.18 & \pm 4.42 & \pm 4.49 & \pm 4.13 \\
\hline & $\mathrm{PDC}$ & -64.68 & -42.54 & -36.79 & -35.14 & -32.35 & -30.47 & -27.76 & -25.87 \\
\hline
\end{tabular}

Table 4

Levels of Antioxidant Enzymes and Products in Liver tissue of Control and Experimental rats. 


\begin{tabular}{|c|c|c|c|c|c|c|c|c|c|}
\hline Parameters & Control & Cd treated & Vit-C & Zinc & NAC & C+Zinc & $\mathrm{C}+\mathrm{NAC}$ & Zinc+ NAC & $\mathrm{C}+\mathrm{Zinc}+\mathrm{NAC}$ \\
\hline \multirow[t]{3}{*}{ MDA } & $475^{a}$ & $1015^{b}$ & $885^{b, c}$ & $901^{b, c}$ & $950^{b, c}$ & $809^{b, c}$ & $804^{b, c}$ & $775^{b, c}$ & $670^{b, c}$ \\
\hline & \pm 25 & \pm 39 & \pm 29 & \pm 40 & \pm 32 & \pm 29 & \pm 32 & \pm 29 & \pm 32 \\
\hline & PDC & +114 & +86 & +90 & +100 & +70 & +69 & +64 & +41 \\
\hline \multirow[t]{3}{*}{ SOD } & $66.79^{a}$ & $31.73^{b}$ & $34.44^{b, c}$ & $36.18^{b, c}$ & $38.14^{b, c}$ & $40.12^{b, c}$ & $42.14^{b, c}$ & $44.75^{b, c}$ & $51.14^{b, c}$ \\
\hline & \pm 2.15 & \pm 1.14 & \pm 1.18 & \pm 1.15 & \pm 1.19 & \pm 1.28 & \pm 1.29 & \pm 1.32 & \pm 1.12 \\
\hline & PDC & -35 & -48 & -46 & -43 & -40 & -37 & -33 & -23 \\
\hline \multirow[t]{3}{*}{ CAT } & $88.14^{\mathrm{a}}$ & $42.44^{b}$ & $44.75^{b, c}$ & $47.75^{b, c}$ & $49.75^{b, c}$ & $53.72^{b, c}$ & $59.14^{b, c}$ & $60.19^{b, c}$ & $68.13^{b, c}$ \\
\hline & \pm 2.12 & \pm 1.13 & \pm 1.14 & \pm 1.25 & \pm 1.32 & \pm 1.34 & \pm 1.42 & \pm 1.38 & \pm 1.18 \\
\hline & PDC & -52 & -49 & -46 & -43 & -40 & -37 & -33 & -23 \\
\hline \multirow[t]{3}{*}{ MPO } & $5.14^{a}$ & $9.85^{b}$ & $8.35^{b, c}$ & $8.19^{b, c}$ & $7.74^{b, c}$ & $7.15^{b, c}$ & $6.74^{b, c}$ & $6.34^{b, c}$ & $6.15^{b, c}$ \\
\hline & \pm 0.28 & \pm 0.38 & \pm 0.32 & \pm 0.31 & \pm 0.26 & \pm 0.25 & \pm 0.27 & \pm 0.26 & \pm 0.24 \\
\hline & PDC & +92 & +63 & +59 & +51 & +39 & +31 & +23 & +20 \\
\hline \multirow[t]{3}{*}{$\mathrm{GP}_{\mathrm{x}}$} & $8.15^{a}$ & $4.05^{b}$ & $4.18^{b, c}$ & $4.34^{b, c}$ & $4.75^{b, c}$ & $5.25^{b, c}$ & $5.79^{b, c}$ & $6.42^{b, c}$ & $7.04^{b, c}$ \\
\hline & \pm 0.46 & \pm 0.28 & \pm 0.24 & \pm 0.25 & \pm 0.26 & \pm 0.29 & \pm 0.32 & \pm 0.35 & \pm 0.28 \\
\hline & PDC & -50 & -49 & -47 & -42 & -36 & -29 & -22 & -14 \\
\hline \multirow[t]{3}{*}{ GSH } & $4.93^{a}$ & $2.85^{b}$ & $2.92^{b, c}$ & $2.94^{b, c}$ & $3.02^{b, c}$ & $3.14^{b, c}$ & $3.25^{b, c}$ & $3.32^{b, c}$ & $3.49^{b, c}$ \\
\hline & \pm 0.41 & \pm 0.22 & \pm 0.23 & \pm 0.24 & \pm 0.27 & \pm 0.29 & \pm 0.32 & \pm 0.33 & \pm 0.32 \\
\hline & PDC & -42 & -41 & -40 & -39 & -36 & -34 & -33 & -29 \\
\hline \multicolumn{10}{|c|}{$\begin{array}{l}\text { Values are expressed as Mean } \pm \text { SD of six individual observations. a,b,c Values are not sharing a common superscript letter (a,b,c) } \\
\text { differ significantly at (P<0.05(OMRT)). PDC: Percept Deviation over Control. MDA: } \mu \text { moles/g wet wt of tissue. SOD: Superoxide } \\
\text { dismutase Units/g wet weight of tissue/minute. CAT: Catalase activity } \mu \text { moles of } \mathrm{H}_{2} \mathrm{O}_{2} \text { degladed/ } \mathrm{g} \text { wet wt of tissue/ minute. } \\
\text { MPO: } \mu \text { moles of maloaldehyde formed / g wet weight. GPX: Glutathione peroxidase nano moles of NADPH oxidized/ mg } \\
\text { protein/ minute. GSH: Glutathione nano moles/g wet weight of tissue. }\end{array}$} \\
\hline
\end{tabular}

Table 5

Levels of Antioxidant Enzymes and Products in Kidney tissue of Control and Experimental rats. 


\begin{tabular}{|c|c|c|c|c|c|c|c|c|c|}
\hline Parameters & Control & Cd treated & Vit-C & Zinc & NAC & $C+$ Zinc & $\mathrm{C}+\mathrm{NAC}$ & Zinc+ NAC & $\mathrm{C}+\mathrm{Zinc}+\mathrm{NAC}$ \\
\hline \multirow[t]{3}{*}{ MDA } & $528^{a}$ & $1114^{b}$ & $1074^{\mathrm{b}, \mathrm{c}}$ & $995^{b, c}$ & $880^{b, c}$ & $740^{b, c}$ & $730^{b, c}$ & $722^{b, c}$ & $685^{b, c}$ \\
\hline & \pm 29 & \pm 35 & \pm 38 & \pm 24 & \pm 22 & \pm 25 & \pm 26 & \pm 27 & \pm 24 \\
\hline & PDC & +111 & +103 & +88 & +67 & +40 & +38 & +37 & +30 \\
\hline \multirow[t]{3}{*}{ SOD } & $45.72^{\mathrm{a}}$ & $24.12^{b}$ & $26.19^{b}$ & $28.12^{b}$ & $30.12^{b, c}$ & $31.18^{b, c}$ & $32.18^{b, c}$ & $33.11^{b, c}$ & $33.75^{b, c}$ \\
\hline & \pm 2.14 & \pm 1.10 & \pm 1.12 & \pm 1.14 & \pm 1.15 & \pm 1.14 & \pm 1.14 & \pm 1.14 & \pm 1.71 \\
\hline & PDC & -47 & -43 & -38 & -34 & -32 & -30 & -28 & -26 \\
\hline \multirow[t]{3}{*}{ CAT } & $82.18^{a}$ & $43.42^{b}$ & $45.45^{b}$ & $48.74^{b}$ & $51.23^{b}$ & $53.42^{b, c}$ & $58.41^{\mathrm{b}, \mathrm{c}}$ & $60.79^{b, c}$ & $65.77^{b, c}$ \\
\hline & \pm 2.12 & \pm 1.14 & \pm 1.13 & \pm 1.14 & \pm 1.24 & \pm 1.42 & \pm 1.56 & \pm 1.57 & \pm 2.12 \\
\hline & PDC & -47 & -45 & -41 & -38 & -35 & -29 & -26 & -20 \\
\hline \multirow[t]{3}{*}{ MPO } & $4.73^{a}$ & $8.73^{b}$ & $8.14^{b, c}$ & $8.04^{b, c}$ & $7.34^{b, c}$ & $7.49^{b, c}$ & $6.43^{b, c}$ & $6.05^{b, c}$ & $5.79^{b, c}$ \\
\hline & \pm 0.24 & \pm 0.42 & \pm 0.33 & \pm 0.32 & \pm 0.29 & \pm 0.28 & \pm 0.31 & \pm 0.25 & \pm 0.23 \\
\hline & PDC & +85 & +72 & +70 & +55 & +58 & +36 & +28 & +22 \\
\hline \multirow[t]{3}{*}{$\mathrm{GP}_{\mathrm{x}}$} & $7.32^{a}$ & $3.92^{b}$ & $4.14^{b}$ & $4.54^{b}$ & $4.89^{b, c}$ & $5.12^{b, c}$ & $5.34^{b, c}$ & $5.59^{b, c}$ & $6.13^{b, c}$ \\
\hline & \pm 0.38 & \pm 0.24 & \pm 0.24 & \pm 0.25 & \pm 0.26 & \pm 0.26 & \pm 0.25 & \pm 0.24 & \pm 0.25 \\
\hline & PDC & -46 & -43 & -38 & -33 & -30 & -27 & -24 & -16 \\
\hline \multirow[t]{3}{*}{ GSH } & $4.12^{\mathrm{a}}$ & $2.42^{b}$ & $2.63^{b, c}$ & $2.79^{b, c}$ & $2.84^{b, c}$ & $3.02^{b, c}$ & $3.12^{b, c}$ & $3.23^{b, c}$ & $3.38^{b, c}$ \\
\hline & \pm 0.34 & \pm 0.22 & \pm 0.22 & \pm 0.21 & \pm 0.23 & \pm 0.23 & \pm 0.24 & \pm 0.24 & \pm 0.24 \\
\hline & PDC & -41 & -36 & -32 & -31 & -27 & -24 & -22 & -18 \\
\hline \multicolumn{10}{|c|}{ 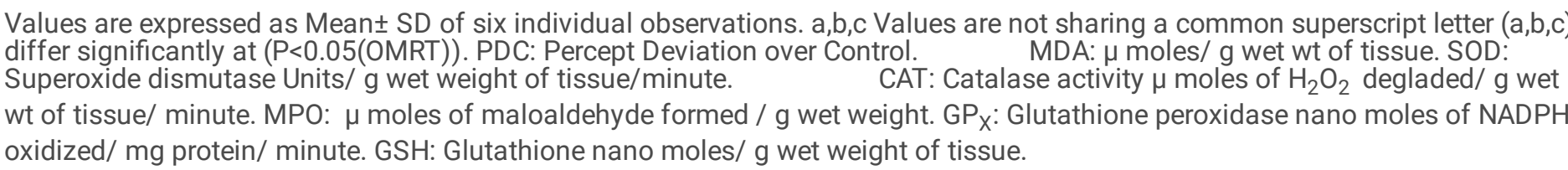 } \\
\hline
\end{tabular}

\section{Supplementary Files}

This is a list of supplementary files associated with this preprint. Click to download.

- GraphicalAbstract.docx 